\title{
Musiques et charismes chez les chrétiens en Égypte au début du XXI ${ }^{\mathrm{e}}$ siècle
}

L'exemple catholique

Music and Charisma Among the Christians in Early $21^{\text {st }}$ Century Egypt. The Catholic Case

Músicas y carismas entre los cristianos en Egipto a principios del siglo XXI. El ejemplo católico

\section{Séverine Gabry-Thienpont}

\section{(2)enEdition} Journals

Édition électronique

URL : http://journals.openedition.org/assr/27108

DOI : 10.4000/assr.27108

ISSN : $1777-5825$

Éditeur

Éditions de l'EHESS

\section{Édition imprimée}

Date de publication : 1 septembre 2015

Pagination : 187-207

ISBN : 9-782713224706

ISSN : 0335-5985

\section{Référence électronique}

Séverine Gabry-Thienpont, " Musiques et charismes chez les chrétiens en Égypte au début du XXIe siècle ", Archives de sciences sociales des religions [En ligne], 171 | 2015, mis en ligne le 01 septembre 2018, consulté le 03 mai 2019. URL : http://journals.openedition.org/assr/27108 ; DOI : 10.4000/ assr.27108 


\section{Séverine Gabry-Thienpont}

\section{Musiques et charismes chez les chrétiens en Égypte au début du $\mathrm{XXI}^{\mathrm{e}}$ siècle}

\section{L'exemple catholique}

Seigneur, dans les discothèques, dans les concerts, on lève les mains, on chante, on danse. Et toi, tu es la plus grande star, donc faisons de même pour toi ! Steven, Égyptien de confession grecque catholique, 24 ans.

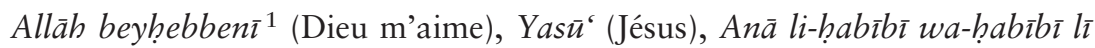
(Je suis à mon bien-aimé et mon bien-aimé est à moi, tiré du Cantique des Cantiques). Ces inscriptions ornent les plaques de gourmettes imitant le bois, attachées à des bracelets en faux cuir sobrement colorés. Exposés sur une table à la sortie de l'église Kanīsat Allāh de Choubra ${ }^{2}$, face à l'imposant escalier permettant l'accès à l'église, les bracelets se vendent facilement. Pendant que les acheteurs potentiels font leur choix, les chants du murannim ${ }^{3}$ Māhir Fāyiz retentissent de la porte ouverte de l'église : la réunion n'est pas encore terminée. Et comme tous les samedis soirs, coptes-catholiques, orthodoxes et protestants occupent les bancs de l'église et bondent les travées. Tous chantent sans exception, le regard tourné vers les écrans où défilent les paroles des tarnìm-s ${ }^{4}$, parfois les bras levés pour mieux se rapprocher de Dieu avec, bien souvent, un sourire extatique aux lèvres.

En Égypte, l'influence des évangéliques et des communautés charismatiques catholiques sur les pratiques religieuses coptes est manifeste. Encore peu étudiée, voire mal identifiée, cette tendance représente une "contre-conduite " - telle qu'entendue par Michel Foucault - à l'orthodoxie copte et dévoile peu ou prou l'ambition d'une renaissance, déjà en marche, dont le recours à la musique se

1. Phrase écrite en dialecte égyptien.

2. Quartier nord du Caire, à forte concentration chrétienne.

3. Chanteur ou compositeur de cantiques, nommés tarnìm-s (S. Gabry-Thienpont, 2014a).

4. Les tarnìm-s sont des chants à argument religieux aux inspirations musicales diverses, interprétés en contexte paraliturgique (S. Gabry-Thienpont, 2014b : 282-283). 
révèle être l'un des principaux vecteurs. Le présent article ambitionne de comprendre la place de la musique dans ces mouvements à travers l'étude de cas de deux groupes catholiques : la Communauté de l'Emmanuel (Mağmū'a 'Immānū'îl) et le Chemin Néocatéchuménal (Ṭariq al-Maw'īziñn al-Ğadìd), tous deux représentés au sein de la capitale égyptienne. Les prémices du Renouveau charismatique en Égypte et l'histoire de l'implantation cairote de ces deux groupes précèderont une étude plus spécifiquement musicale de leurs pratiques, associée à une mise en perspective avec les musiques composées par la Praise Team of Egypt, Al-Kārūz et Better Life, groupes issus de la mouvance évangélique. Ce travail permettra de nourrir notre compréhension des interactions à l'œuvre entre protestants, catholiques et orthodoxes, à l'heure de ce qui commence à être perçu, dans le giron même de l'Église copte, comme un renouveau dans le Renouveau.

\section{Petite genèse de l'implantation du Renouveau charismatique en Égypte}

Issu d'Amérique du Nord, le Renouveau charismatique rencontre un succès très net en Europe dès le début des années 1970, à la suite de la mise en place du II concile œcuménique du Vatican (1962-1965). Les congrégations religieuses européennes qui se sont succédé en Égypte depuis le XVI siècle (Hamilton, 2006 : 58) ont joué un rôle considérable dans l'implantation progressive du mouvement dans ce pays. Dès la fin des années 1970, il pénètre Le Caire, rencontrant un succès cette fois progressif, mais durable, auprès des multiples confessions chrétiennes représentées dans la capitale.

\section{« Culture » européenne et Renouveau catholique}

En Égypte, les chrétiens catholiques sont représentés par plusieurs obédiences et en proportions diverses : coptes-catholiques, melkites, syriens, arméniens, chaldéens, maronites et latins. Des missionnaires jésuites et franciscains sont à l'origine des premières conversions d'Égyptiens vers le catholicisme, qui datent vraisemblablement de la toute fin du XVII ${ }^{\mathrm{e}}$ siècle (Id.: 82). En 1895, l'Église copte-catholique est officiellement unie à Rome par la création d'un patriarcat. Cette Église, à la fois nationale - car copte - et importée - car catholique, constitue aujourd'hui l'essentiel de la présence catholique en Égypte. Dès l'origine, l'influence des missionnaires s'est caractérisée par la diffusion, à travers l'éducation, d'une culture européenne prédominante. Il en est d'ailleurs de même chez les évangéliques (Sharkey, 2008 ; Sedra, 2011).

Les enquêtes que nous avons menées en 2014 et 2015 au sein des communautés catholiques de la capitale révèlent que le mouvement charismatique ne touche pour l'instant qu'une minorité sociale, généralement scolarisée dans des écoles privées d'origine européenne, toujours congréganistes, ou fréquentant des paroisses 
latines. La grande majorité des personnes rencontrées connaissent l'anglais, voire le français - langues d'enseignement privilégiées dans ces écoles -, côtoient de grandes universités, comme l'Université américaine du Caire, partent étudier en Europe, au Canada, aux États-Unis. Occidentalisés et polyglottes, les membres des communautés catholiques en Égypte semblent s'approprier aisément ces pratiques communautaires d'origine européenne.

Avant d'être introduit en Égypte, le Renouveau charismatique apparaît en Europe après le concile Vatican II. Pour le qualifier à ses débuts, les expressions variées de "Charismatic Renewal », « Mouvement catholique néo-pentecostal », "Renouveau dans l'Esprit » ou encore, «Renouveau spirituel » ont tour à tour été adoptées, puis abandonnées (Laurentin, 1974 : 7). Cette difficulté à qualifier le mouvement ${ }^{5}$ s'explique par l'adhésion à celui-ci de cercles d'obédiences très diverses. Et comme le note Jean-Paul Willaime à propos du pentecôtisme, le « foisonnement et [...] la plasticité des groupes » (Willaime, 1999: 5) ne rendent pas aisée l'identification des différentes communautés qui s'en réclament, ni leurs particularités.

D’abord, le Renouveau charismatique, également désigné de nos jours, notamment par Olivier Landron, par l'expression « Renouveau communautaire postconciliaire ", hérite du pentecôtisme, lui-même héritier du méthodisme dans son approche, de l'idée d'une rencontre personnelle avec Dieu (Pina, 2001: 26). Dans sa volonté de renouvellement de l'Église catholique, le concile Vatican II avait alors appelé "à une nouvelle Pentecôte sur le monde, une Pentecôte d'amour " ${ }^{6}$ (Peyrous, in Goursat, 2011 : 9). L'inspiration des mouvements pentecôtistes du début du XX $\mathrm{XX}^{\mathrm{e}}$ siècle pour le renouvellement liturgique souhaité par les catholiques est en effet évidente, notamment par la place accordée aux dons de l'Esprit saint, les charismes ${ }^{7}$.

La reconnaissance de ces charismes devient indispensable pour renouveler sa foi et se " convertir ». Par « conversion », les catholiques - comme les protestants - entendent aussi bien leur rencontre avec le Christ qu'avec Dieu ou avec le Saint-Esprit. L'expression nord-américaine pentecôtiste de Born Again Christian - également connue sous celle de mawlūd min ğadīd dans certains pays arabes

5. L'intérêt d'approcher le Renouveau charismatique comme un mouvement est un parti pris explicité par Thomas J. Csordas en ces termes : "I examine the Renewal as a "movement", arguing that this is an obvious but also a problematic theoretical category under which to subsume the phenomenon, and introducing a distinction between religions of peoples and religions of the self »(Csordas, 1997 : préface).

6. Fête chrétienne qui commémore la descente de l'Esprit saint sur les Apôtres, la Pentecôte a lieu cinquante jours après Pâques. Cette recherche d'une nouvelle Pentecôte, où l'Esprit saint descend sur les laïcs qui sont prêts à le recevoir, représente le fil conducteur de la création des communautés charismatiques.

7. Ces dons sont présentés dans le Nouveau Testament, plus précisément dans les Actes des Apôtres (guérisons miraculeuses, glossolalie, etc.) et dans les écrits de Paul de Tarse. 
comme au Liban, mais peu usitée en Égypte - prend tout son sens pour désigner ces charismatiques catholiques, qui se démarquent néanmoins rapidement du Pentecôtisme par leur attachement au septénaire sacramentaire et l'importance qu'ils accordent à la piété mariale.

Pour permettre cette conversion, l'engagement individuel est nécessaire, en tant qu' "expérience mentale, intime, privée », pour reprendre les mots de l'anthropologue Albert Piette lorsqu'il explicite la notion de «croire» (2014 : 63). Et c'est précisément au sein de cet engagement individuel que les laïcs jouent un rôle fondamental. Après avoir reçu le «baptême dans l'Esprit saint » - ou effusion de l'Esprit - par imposition des mains, ils prennent le parti d'œuvrer activement à la revitalisation de l'Église catholique. Communautés et groupes de prière naissent alors dans cette dynamique. Cela signifie que des laïcs décident de se rassembler régulièrement et de partager un même idéal religieux, dont l'accomplissement passe par des règles communes, propres à chacun de ces groupes ou communautés. En effet, dans ce Renouveau, toute la production, si l'on peut dire, des démonstrations de la foi, est repensée. C'est par exemple dans ce contexte que de nombreux jeunes sans formation liturgique particulière, voire sans « enracinement ecclésial profond »(Landron, 2004 : 34), manifestent un goût certain pour de nouvelles compositions musicales et le développent.

En Égypte, en tant que minorité dans un pays où l'islam est religion d'État, les catholiques attachent beaucoup d'importance aux rassemblements communautaires, au même titre que les coptes-orthodoxes qui se réunissent bien souvent dans l'enceinte des églises pour y accomplir diverses activités. Celle de l'église des missionnaires comboniens du Cordi Jesu, au centre du Caire, par exemple, est agrémentée d'un bar, de tables et de chaises, ainsi que de salles où se réunissent les membres du Chemin Néocatéchuménal dont il sera question plus loin. En Égypte, le Renouveau charismatique, de même que le Renouveau copte ${ }^{8}$, prend appui sur ce goût pour l'entre-soi communautaire, et le renforce.

\section{La Communauté de l'Emmanuel et du Chemin Néocatéchuménal au Caire}

Entrer dans une communauté implique d'intégrer un groupe qui s'apparente à une deuxième famille. Prier ensemble, manger ensemble, réfléchir ensemble, s'adresser ensemble à Dieu, se confesser ensemble, constituent autant d'éléments caractéristiques des communautés charismatiques en général et en Égypte en particulier. "Au début, on n’y arrive pas, on ne peut pas [prier à haute voix tous

8. Avancé pour la première fois par Hervé Legrand (1962), le terme de « renouveau » copte permettait d'identifier l'action du clergé dans les années 1960, au sein de l'Église copte (El Khawaga, 1993). Il se manifeste dans les faits par une revalorisation de l'identité copte, un retour aux sources - Église primitive -, une recrudescence des vocations monastiques et une systématisation de l'éducation, notamment musicale (Gabry-Thienpont, 2013). 
ensemble]. Mais quand le déclic se fait, alors on est libéré ». C’est en ces termes que Nadia me résume l'apport inestimable qu'a représenté pour elle la naissance de la Communauté de l'Emmanuel en Égypte. Tout commence au début des années 2000. Une Française, Christine ${ }^{9}$ vit alors au Caire avec son mari. Ellemême fait partie de la Communauté de l'Emmanuel en France. "Au cours des six années où nous étions au Caire, me raconte-elle, et portant les grâces [c'està-dire l'adoration, la compassion et l'évangélisation] de la Communauté de l'Emmanuel, je vivais mon quotidien aussi fidèle que possible à l'Eucharistie, à la charité et à l'adoration ». Christine suit, en parallèle, des cours d'arabe avec un professeur particulier, une copte-catholique. La sixième et dernière année de sa présence au Caire, en 2003, cette dernière lui avoue avoir été « touchée par sa vie » : elle souhaite donc se rendre à Paray-le-Monial, lieu de pèlerinage en France, revivifié dès 1975 par Pierre Goursat (1914-1991), fondateur de l'Emmanuel (Peyrous, Catta, 2006 : 78). Elle pose une autre requête, celle d'organiser une retraite en Égypte sur les "grâces » de l'Emmanuel, et plus particulièrement, sur celles du "Cœur de Jésus ". C'est en effet à partir de Paray-le-Monial que la Compagnie de Jésus propage cette dévotion, fondée sur l'apparition du Cœur à la religieuse sainte Marguerite-Marie Alacoque ${ }^{10}$ et inspirée par les écrits de saint Claude la Colombière (1641-1682), prêtre jésuite français, qui la dirigea. C'est ainsi que la Communauté de l'Emmanuel de Paris envoie au Caire un prêtre arabophone, le père Dominique, et que Christine et un autre "frère " français (selon le terme en usage parmi les membres des communautés charismatiques pour se désigner), mettent en place une retraite de cinq jours. À leur grande surprise, trente-huit participants égyptiens s'y inscrivent alors, puis treize d'entre eux demandent une préparation à l'effusion de l'Esprit. La même année, huit d'entre eux, dont un prêtre copte-catholique, se rendent à Paray-le-Monial pour participer à une session d'été. Ainsi est née la Communauté de l'Emmanuel en Égypte.

Jeune prêtre dynamique, Dominique poursuit pendant quelque temps les retraites en Égypte, une à deux fois par an. Il organise alors régulièrement des marches de quatre à cinq jours avec des pauses de méditation dans le Désert Blanc (désert occidental de l'Égypte), où l'accompagnaient des jeunes à la fois Européens et Égyptiens. Des temps de silence, de marche seul ou en binôme structurent ces retraites. Lui-même membre de l'Emmanuel, le père Dominique lance le premier groupe communautaire à Héliopolis, en 2003. La Communauté ne compte alors que peu de membres à son actif : ils ne sont que cinq. Mais le modèle suivi est le même que celui établi par les communautés françaises de l'Emmanuel : la louange et l'adoration deviennent prépondérants, le principe des

9. Pour des raisons de confidentialité, le prénom a été modifié.

10. Sur la diffusion du culte du Sacré Cœur chez les chrétiens arabes, plus particulièrement, chez les maronites au Liban, ainsi que sur l'affirmation de leur identité catholique, $c f$. Heyberger, 2001. 
"maisonnées ${ }^{11}$ » est repris tel quel, de même que celui des journées communautaires où alternent activités, prières, chants et séminaires. En 2014, la Communauté de l'Emmanuel en Égypte comptait environ soixante-dix membres.

De son côté, le Chemin Néocatéchuménal est une communauté qui a germé dans les bidonvilles de Palomeras, en banlieue de Madrid, au contact de Kiko Argüello. Le Chemin Néocatéchuménal ne représente pas une communauté charismatique au même titre que celle de l'Emmanuel. Les catéchèses de Kiko Argüello ne se focalisent pas sur l'importance des dons de l'Esprit saint, mais sur celle du kérygme. Le kérygme est la profession de foi des chrétiens, qui implique d'énoncer la qualité de Messie du Christ et d'admettre sa résurrection ainsi que son appel à la conversion. Le kérygme de Pierre figure néanmoins dans le Nouveau Testament le jour de la Pentecôte, et interpelle sur le don du Saint Esprit (Ac $2: 22-24,32$ et 38). Le Chemin s'identifie ainsi davantage à un itinéraire d'apprentissage de la foi, tout en inscrivant tout de même son action dans le Renouveau de l'Église catholique postconciliaire. Le rapprochement des deux se justifie également par l'usage du terme de «communauté » par son fondateur lui-même, lorsqu'il explicite le "tripode », essence-même du Chemin (Argüello, 2013 : 59). Enfin, les confluences entre les communautés charismatiques et le Chemin s'éprouvent au fil des exemples, comme nous le verrons, témoignant d'un socle de valeurs communes solide.

La première communauté du Chemin née dans les baraques de Palomeras en 1964, juste avant la fin du Concile Vatican II, était constituée de gitans et de " quinquis ", c'est-à-dire de quincalleros, des quincaillers semi-nomades assimilés à des voyous et à de petits trafiquants. La diffusion du Chemin en dehors de l'Espagne, et d'abord en Italie, fut fulgurante. En 1977, il arrive en Égypte ${ }^{12}$. Cette année-là, le père combonien Camillo Palalin est responsable de l'Église catholique latine Saint-Joseph de Zamalek, quartier aisé du Caire. Dans son article publié dans Le Messager, le père raconte que la plupart des activités menées au sein de la paroisse depuis plusieurs années tendaient à s'interrompre, par manque d'investissement de la part des laïcs, à l'exception de la catéchèse aux enfants. Mais que restait-il donc pour les adultes ? Comment progresser dans la foi ? Un religieux (nāsik) venu d'Italie pour apprendre l'arabe, logeait alors à la paroisse. Il parlait volontiers de la Bible, expliquait comment le Livre Saint pouvait illuminer la vie du peuple de l'Église, et évoqua l'existence du Chemin Néocatéchuménal.

11. La Communauté de l'Emmanuel rassemble des milliers de fidèles. Le principe des « maisonnées " a été fondé pour répartir ces nombreux " frères " au sein de petits groupes, permettant ainsi un cheminement ensemble - ce qui rappelle, nous le verrons, l'organisation des groupes au sein du Chemin Néocatéchuménal. Ces "maisonnées ", dont le nom évoque l'attachement quasi-familial recherché par les communautaires, se tiennent à intervalle hebdomadaire.

12. Les renseignements concernant les débuts du Chemin en Égypte sont tirés de l'article paru dans le journal Le Messager daté du 2 novembre 2003 (n²34), intitulé " Ğamā‘àt Ṭarīq al-Maw'ūzīin al-Ǧadīd fĩ Miṣr munđu 1977 », rédigé par le père combonien Camillo Palalin. 
En décembre 1976, trois prédicateurs (kārazūn) italiens vinrent en Égypte pour se consacrer à la prédication et à l'enseignement du kérygme. "En acceptant de les suivre, écrit-il, on accepte de crier notre foi de manière nouvelle, en faisant un examen de l'amour de Dieu, transmis par Jésus-Christ ». Ces prédicateurs ont ainsi organisé une nouvelle catéchèse (al-maw'üziyya) pour les adultes. En mars 1977, la première communauté du monde arabe est fondée en Égypte. Elle naît à Héliopolis (Miṣr al-Ǧadīda), autre quartier aisé de la capitale, en l'église de Sainte-Thérèse (tenue par des Arméniens catholiques). Puis, en 1981, le groupe de Zamalek s'installe en l'église du Cordi Jesu dans le centre du Caire, tenue par les comboniens depuis le XIX ${ }^{\mathrm{e}}$ siècle. Inaugurée et bénie en 1930, cette église remplaçait une plus ancienne, érigée en 1883 à partir du projet du défunt évêque Daniel Comboni (1831-1881), fondateur de la congrégation des missionnaires comboniens, dévoués au Cœur de Jésus ${ }^{13}$. Quelques prêtres comboniens résident encore aujourd'hui dans les bâtiments adjacents à l'église, et certains dirigent même parfois les offices pour les membres du Chemin, si ce dernier n'a pas de prêtre membre présent, ce qui est normalement requis. Depuis 1981, les groupes du Cordi Jesu et d'Héliopolis du Caire se sont considérablement agrandis. Toutes les obédiences chrétiennes présentes au Caire - pas seulement catholiques, donc y sont représentées. Des groupes évoluent également à Alexandrie, au Liban, en Jordanie, en Palestine, au Soudan, et en Irak. En 2003, le patriarche copte catholique, le patriarche arménien catholique et l'évêque des maronites ont demandé que la langue arabe soit en usage dans les communautés du Chemin dans le monde arabe, incitant dorénavant à la traduction systématique des catéchèses et des chants de la communauté dans cette langue.

En 2014, vingt-trois Chemins, c'est-à-dire vingt-trois groupes de prière, sont répartis au Caire dans cinq paroisses. Six de ces groupes évoluent en l'église du Cordi Jesu. Une trentaine de personnes en moyenne vivent un Chemin ensemble, ce qui signifie qu'elles reçoivent une même catéchèse. Le système du Chemin fonctionne par niveau catéchétique : ceux qui commencent un cheminement ensemble le poursuivent toujours ensemble jusqu'à son terme. En 1995, l'actuel responsable du Chemin en Égypte, Na'meh, a débuté avec son propre groupe, toujours uni. Le Chemin est aussi un chemin de conversion ${ }^{14}$ : parvenir définitivement à aimer son ennemi, grâce à l'Esprit Saint, en adéquation avec le « serment sur la montagne » (Évangile selon Matthieu) ${ }^{15}$, signifie que le Chemin est terminé. En 2015, Na'meh et son groupe parvenaient au bout de ce cheminement.

13. Revue missionnaire «Le Sacré-Cœur et la mission de l'Afrique centrale ", Janvierfévrier-mars 1930, Collège de la Sainte Famille, Mélanges Égypte chrétienne contemporaine, v.

14. Sur un christianisme de conversion tel qu'entendu par les protestants et réapproprié par certains prêtres coptes orthodoxes, $c f$. Du Roy, 2014 : 353-358.

15. Et en particulier avec le verset suivant : "Aimez vos ennemis, bénissez ceux qui vous maudissent, faites du bien à ceux qui vous haïssent, et priez pour ceux qui vous maltraitent et qui vous persécutent " (Mt, 5-37). 
Dans les deux cas, l'action d'un individu a constitué l'élément déclencheur de l'implantation de ces communautés en Égypte. Les membres de l'Emmanuel et du Chemin se considèrent en effet comme des évangélisateurs. Et c'est du reste là l'une des tâches qui leur a été confiée par les fondateurs des deux communautés, Pierre Goursat (2011) et Kiko Argüello (2013). Chaque frère évolue donc, non pas dans une foi individuelle hermétique à l'extérieur, mais en interaction quasi-permanente avec cet extérieur - d'où le terme même de "communauté ", impliquant un partage commun de valeurs et de convictions. Christine a témoigné auprès de ses amis proches égyptiens enclins à l'écouter. Elle leur a raconté la Communauté, a expliqué ses valeurs, ses engagements, ce qu'elle estimait être une force et une manière salvatrice de vivre la foi, de l'exprimer. Comme nous l'avons vu, c'est un religieux venu d'Italie, où le Chemin était bien implanté, qui, faisant le constat de défaillances dans l'activité pastorale de la paroisse catholique de Saint-Joseph, décide de prêcher le kérygme d'abord au prêtre de la paroisse, puis aux fidèles désireux de l'écouter. La proximité culturelle entre l'Occident et les catholiques égyptiens, du fait de l'enseignement et de l'encadrement des fidèles par des religieux et des missionnaires européens, a indubitablement facilité l'implantation de ces communautés au Caire. Aujourd'hui en Égypte, le Chemin Néocatéchuménal rassemble bien plus de membres que l'Emmanuel, dont le succès dans le monde arabe est beaucoup plus tardif. Les deux organisations ont la volonté de s'étendre à la Haute-Égypte, mais en dépit de certaines tentatives, elles n'y ont pas réussi pour le moment.

\section{Louange, adoration, parole rythmée... La musique en action}

Au sein de la Communauté de l'Emmanuel et du Chemin Néocatéchuménal, comme dans toutes les communautés postconciliaires du reste, la musique est omniprésente. Indispensable, elle représente une manifestation privilégiée de la piété des «frères» $(a l$-ib̧wa). À travers les différents usages de la musique, on peut observer que les interactions entre les membres des communautés, leur environnement musical, leur environnement sonore et leurs référents culturels sont multiples et complexes, sans compter les modalités expressives relatives à l'une et l'autre de ces interactions. Si, à travers un travail qui reste encore à mener, ces dernières étaient clairement identifiées, cela constituerait un apport inestimable à la compréhension des influences et des processus à l'œuvre dans la composition et la réception de ces chants communautaires. Nous pouvons néanmoins d'ores et déjà nous interroger sur l'importance donnée aux chants communautaires dans l'expression de la foi religieuse des " frères ", ainsi que sur les aspirations dont elles témoignent. 


\section{«L'Église ne se renouvellera pas avec des guitares »}

Vendredi 6 juin 2014, 10 h, école du Sacré-Cœur à Héliopolis. Les membres de la Communauté de l'Emmanuel arrivent au compte-goutte et se saluent avec force embrassades et chaleureuses accolades. Dans leur écrasante majorité, ils sont jeunes, âgés de vingt à trente ans tout au plus. Magdi et Nadia, les deux "patriarches ", comme les principaux concernés aiment se nommer, les accompagnent. Ils étaient là avec Christine, aux débuts de l'Emmanuel en Égypte en 2003. Dans la même enceinte, une église est accolée à l'école avec, autour d'elle, un jardin verdoyant et entretenu. C'est là que la vingtaine de jeunes "frères " présents se réunissent pour débuter cette "journée comm' », comme ils disent. La plupart, parfaitement francophones ou anglophones, glissent fréquemment des mots de ces deux langues dans leurs discours, surtout lorsqu'il s'agit de mots propres à l'Emmanuel : puisque cette communauté est originaire de France, les termes caractéristiques sont toujours désignés en français. L'adoration, par exemple, en arabe suǧŭd, est toujours nommée " adoration », quand bien même celui qui s'exprime le fait en arabe : anâ 'âmel adoration, "je fais l'adoration ". La «journée comm' » débute donc par une louange en plein air. Des requêtes individuelles alternent avec des chants, tirés du livret " Il est vivant ! », répertoire musical propre à la Communauté de l'Emmanuel. Ces chants sont exclusivement des compositions en français, sur des mélodies composées en France, traduits ici en arabe dans le contexte égyptien. Seul un cantique interprété pendant ce rassemblement extérieur a été composé par les membres égyptiens de l'Emmanuel, désireux d'injecter un peu d' " égyptianité » au mouvement.

En général, les rythmes et les échelles des chants de l'Emmanuel sont très différents de ce qu'on entend à la radio égyptienne. Un des enjeux devient donc de leur donner une empreinte arabe, orientale. Cet objectif s'est concrétisé dans le troisième et dernier album enregistré par l'Emmanuel en Égypte : si dans le deuxième disque les chants ont été simplement réarrangés, dans le troisième, figurent de nouvelles compositions aux textes et aux mélodies écrits par des Égyptiens. La terminologie est la même que celle des chants de «Il est vivant ! » : gloire du Seigneur, amour du Christ... Mais les textes représentent davantage des méditations ou des chants d'adoration, inspirés de versets des évangiles, que de la louange. Dans les clips de l'Emmanuel, tournés par les membres en Égypte, l'égyptianisation du répertoire passe également par l'arrangement musical : des instruments comme le qanūn et le nay sont alors utilisés, apportant avec eux leurs caractéristiques techniques et modales ${ }^{16}$. Ce désir d'orientaliser revient fréquemment dans les discussions : à la fin de la journée communautaire, pendant les annonces, la responsable du groupe a par exemple rappelé que la Communauté venait certes de France, mais que l'Église qui leur permettait de vivre leur foi est

16. En voici un exemple : https://www.youtube.com/watch?v=ecMRNQofIrA, consulté le 5 avril 2015 . 
l'Église copte-catholique, et que c'est elle qui marque leur lien avec le Vatican elle requérait dès lors la présence des membres pour animer une messe chez les coptes-catholiques à Héliopolis, à la demande du prêtre de la paroisse, quelques jours plus tard. Cette remarque rappelle la propre analyse de Maurice Martin lorsqu'il conclut son article sur les coptes-catholiques en ces termes:

En fin de compte se manifeste ici le double défi que doit relever la communauté copte catholique pour être fidèle à sa vocation originelle : sauvegarder l'apport à l'Égypte de son intégration à un catholicisme universel adapté au monde d'aujourd'hui, tout en restant fidèle au christianisme de tradition égyptienne pour communiquer ses richesses au catholicisme. Sans privilégier l'une ou l'autre de ses attaches ni jouer leur alternance (Martin, 1990 : 55).

En Égypte, pour les frères des nouvelles communautés, l'enjeu est en effet de signifier de manière explicite leur lien avec une église égyptienne, donc nationale. En l'occurrence, celle des coptes-catholiques. Même si l'origine du Renouveau charismatique est occidentale et que celle de l'Église copte-catholique l'est tout autant, les fidèles font valoir avec une certaine insistance leur identité égyptienne.

La plupart des membres montre une aisance étonnante à s'approprier les tarnìm-s : ils tapent dans leurs mains des rythmes bien plus sophistiqués que ne le serait une simple pulsation, chantent d'autres voix, c'est-à-dire des lignes composées à partir de la structure harmonique du chant, voire produisent des contre-chants très justes. Cette maîtrise musicale, qui leur permet de s'investir pleinement dans la démonstration chantée, témoigne d'une éducation de l'oreille approfondie (extrait sonore ${ }^{17}$ ). Ces jeunes gens sont en outre capables d'improviser tous ensemble des sons et des harmonies sur des voyelles, rappelant ainsi l'attachement de Pierre Goursat pour le «chanter en langues " (Peyrous, Catta, 2006 : 55 ; extrait sonore $\left.{ }^{18}\right)$. L'un des dons de l'Esprit saint serait en effet le " parler en langues », nommé glossolalie. Pour Pierre Goursat, il peut s'exprimer par le chant, libérant ainsi les fidèles de tout carcan linguistique et musical en laissant libre cours au chant glossolale. Cette caractéristique n'est pas propre à l'Emmanuel : Valérie Aubourg décrit en effet une pratique chantée similaire, observée dans un groupe de prière catholique à La Réunion (Aubourg, 2014 : 255-258). Pratiqué au sein de la Communauté en Égypte, le chant glossolale « en langues » prend la tournure d'une improvisation collective inspirée d'un chant précédemment chanté. Le chemin harmonique est, dans ce cas, encore présent dans les oreilles des uns et des autres. Il constitue une base sur laquelle l'improvisation peut se mouvoir en des voyelles indistinctes et où chacun trouve alors intuitivement des repères auditifs. Ce chant glossolale se pratique essentiellement durant l'Adoration du Saint Sacrement.

L'importance de la musique au sein de la Communauté de l'Emmanuel est telle qu'elle occupe une place fondamentale dans l'école d'évangélisation implantée

17. Extrait sonore : https://archive.org/details/AlleluiaCommunauteDeLEmmanuelHeliopolis

18. Extrait sonore $:$ https://archive.org/details/AdorationImproCollective 
en Allemagne. Celle-ci est une des quatre écoles dirigées par la Communauté de l'Emmanuel, la première ayant été fondée par Pierre Goursat en 1984 (Peyrous, Catta, 2006 : 167). Elles sont installées à Paray-le-Monial (France), Rome (Italie), Altötting (Allemagne) et Manille (Philippines). Elles portent depuis peu toutes le même nom, l'ESM (Emmanuel School of Mission). Mais il y a cinq ans, ces écoles étaient encore désignées différemment les unes des autres. Ainsi, celle d'Allemagne s'appelait l'Internationale Akademie für Musik (IAM), ce qui manifestait clairement cet attachement à la formation musicale à des fins évangélisatrices, sur lequel l'école mettait l'accent en prenant prétexte de la profonde imprégnation de la musique religieuse dans la culture allemande. La formation durait un an, et c'est encore le cas aujourd'hui. Quand Steven y participa, il monta avec d'autres une comédie musicale sur Marie-Madeleine, scénographiée en deux temps : selon la Bible et selon le temps actuel. Tous les élèves descendaient également régulièrement dans la rue pour des séances de street music, agrémentées de chorégraphies. Ces séances se tenaient devant les églises et dans les espaces publics, et visaient à inciter les chalands à assister à l'adoration ou à des veillées : "la musique parle, la chorégraphie aussi, raconte-il, donc les gens écoutent beaucoup plus ». Leurs chants étaient entrecoupés d'un petit témoignage rapide de quelques minutes expliquant le changement - entendre ici, la conversion, dans le sens de Born Again -, la louange, la découverte des Évangiles. Puis la musique reprenait.

Aujourd'hui, de même qu'à l'époque, des cours de chant sont dispensés par cette école d'évangélisation. Les étudiants répètent beaucoup les chants de l'Emmanuel avec des groupes de musique rock, créant ainsi du «Christian Rock ", pour reprendre leur expression. Plusieurs membres de la Communauté de l'Emmanuel du Caire ont suivi la formation proposée dans l'une des quatre écoles d'évangélisation. Chacun en garde un souvenir enthousiaste et ému.

Samedi 7 juin 2014, 20 h. Église du Cordi Jesu au centre du Caire. Autre date, autre lieu, autre communauté. Le Chemin Néocatéchuménal célèbre à cette heure tardive la messe de la Pentecôte. Cette fête est d'autant plus importante qu'elle commémore la descente de l'Esprit Saint - dont on connaît l'importance pour les charismatiques - sur les Apôtres. À cette occasion, comme chaque année, les nouveaux membres du Chemin sont accueillis par une danse festive mais sobre, au terme de l'office. Tous sont alors réunis en un immense cercle et tournent au son d'un chant accompagné par la guitare, frappant dans les mains le rythme " deux croches-noire » en continu et évoluant en un pas de côté à peine sophistiqué, précisément répété par tous. Comme dans les réunions de prière des groupes, l'office présente des lectures de la Bible, des chants propres au Chemin - traduits en arabe -, des partages d'expériences après chaque lecture - un membre prend alors librement la parole en se mettant debout et en parlant à voix haute, ce qui pourrait s'apparenter à une sorte de confession publique - et une communion eucharistique. Chacun tient alors l'hostie dans sa main, attendant que tous l'aient 
reçue, avant de la porter à sa bouche, ensemble. Puis la coupe de vin est offerte par un membre et chacun boit une gorgée. Ces éléments composant l'office célébré au sein du Chemin doivent être conservés quelle que soit la paroisse dans laquelle est accompli le rite, car en Égypte, les membres du Chemin ne suivent pas la messe systématiquement dans les mêmes églises. À ce titre, l'une des particularités de cette communauté du Proche-Orient se révèle dans la proximité avec les divers rites orientaux. Si le Chemin suit la messe dans une église syriaque, alors elle en épousera le rite. S'il s'agit d'une église copte-catholique, c'est la messe copte-catholique qui sera suivie. L'eucharistie et le partage des expériences sont propres au Chemin, ce que la communauté doit conserver dans n'importe quelle paroisse, mais la messe et les textes dépendent du rite de l'église d'accueil. Quant aux musiques, finalement, elles ne sont pas considérées comme telles. D'un point de vue strictement dogmatique, les chants composés par Kiko portent une vocation catéchétique. Chaque texte chanté est tiré de la Bible. Il s'avère donc aussi important que les lectures. Plutôt que de "musique ", les membres du Chemin parlent de « rythme » qui fait « résonner les paroles » ${ }^{19}$. Dans la représentation de ces chants, par exemple, un psaume qualifié de joyeux devra être accompagné d'un rythme vif et entraînant. Les chants du Chemin s'apparentent ainsi à de la parole rythmée. Eu égard à l'importance de ces chants dans le cheminement catéchétique, la participation de l'assemblée doit être totale, ce que la mise en musique de la Parole doit précisément permettre. Beaucoup critiquent en effet la passivité des fidèles au sein des rites orientaux, où les chants sont systématiquement du ressort d'une chorale ou de šammās (diacres). Dans le Chemin, tout le monde participe. Le chant, partie intégrante de la catéchèse, se situe à la base même de la réception de la Parole et s'avère indispensable, à ce titre, au kérygme.

Kiko raconte qu'il est allé vivre dans une baraque de Palomeras « avec une guitare et une Bible " (Argüello, 2013 : 40). Les chants ont donc pour la plupart été composés à partir de cet instrument. Dans bon nombre de ces cantiques, des harmonies simples et répétées sont jouées à la guitare, la main droite grattant les cordes pour faire sonner les accords. Dans les offices, plusieurs guitares sont jouées à tour de rôle ou parfois, toutes ensemble, accompagnées d'une tabla, instrument de percussion très utilisé en Égypte, et d'un tambourin. Le jeu peut également s'illustrer par des accords arpégés - tel l'Ave Maria de Kiko -, si l'ambiance (al-ğaw) recherchée se veut davantage recueillie. Aux couplets des psaumes, chantés par un soliste, l'assemblée de fidèles enchaîne en chœur le refrain. Les processus sont les mêmes dans les groupes de prières hebdomadaires : un guitariste, responsable de la session au même titre que le laïc prêcheur, intervient entre chaque lecture et expression personnelle pour entonner un cantique. Le guitariste est le chanteur : il ne s'agit pas pour lui d'accompagner quelqu'un d'autre, mais bien de s'accompagner lui-même. Dans le Chemin, le guitariste met en musique les textes de la Parole tout en participant à la transmission du kérygme.

19. Na'meh, communication personnelle, 9 juin 2014. 
«L'Église ne se renouvellera pas avec des guitares ». Ainsi parlait Kiko aux adeptes des messes beat ${ }^{20}$ en Italie, créées dans une veine toute woodstockienne. L'intérêt des chants au sein des communautés charismatiques est en effet de véhiculer les préceptes de la foi - évangéliser et catéchiser, donc - par l'entremise d'un média accessible à tous, et plaisant. Si la pratique musicale occupe une place essentielle dans le Renouveau, ce n'est pas la musique en tant que telle qui compte, mais bien ce qu'elle contribue à véhiculer : prière, catéchèse et louange. Ainsi, la Communauté de l'Emmanuel comme le Chemin Néocatéchuménal accomplissent un service d'évangélisation, bien que le mot tašbìr (évangélisation) ne soit pas utilisé en Égypte : l'action d'évangéliser, considérée comme du prosélytisme, est passible d'emprisonnement. Ce service invite à lire et à écouter l'évangile, et le chant en est l'un des outils. Pour les deux communautés, il se concentre sur les chrétiens : le but en soi n'est pas de convertir les musulmans, mais d'aider les chrétiens à vivre leur foi, tout en les incitant à rester dans leurs paroisses. Ce service, manifesté par des événements dans les églises, est ouvert à tous. Les membres de l'Emmanuel appellent cela des «Emmanuel Events » et les publient sur la page Facebook de la Communauté en Égypte. Ils animent alors la louange et l'adoration dans différentes églises. Les membres du Chemin, eux, prêchent régulièrement dans les églises et se rendent chaque année dans les paroisses en octobre pour se présenter et amener à eux de nouveaux membres.

\section{Évangéliques, protestants, charismatiques... Une même expression musicale?}

Chez les évangéliques et autres protestants, les pratiques musicales occupent un rôle tout aussi manifeste dans les églises. Dans les deux cas, on retrouve l'importance des réunions (nommées Bible Studies chez les protestants, car très centrées sur les Écritures) et l'usage des chants pour exprimer la foi et évangéliser. Néanmoins des différences fondamentales persistent, notamment en ce qui concerne le socle de tolérance accordée aux pratiques musicales et corporelles en contexte religieux. O. Landron rappelle à ce sujet que les catholiques charismatiques français ont éprouvé, au milieu des années 1970, un besoin d'organisation au sein du Renouveau, ce qui entraîna la mise en place progressive d'un cadre institutionnel. Pour les pentecôtistes protestants, un tel cadre risquait de conduire à " un "barrage” à la spontanéité des grâces accordées par l'Esprit Saint » (Landron, 2004 : 200), provoquant ainsi une rupture liée à la conception du rôle des chants au sein des réunions. En Europe, les relations entre catholiques pentecôtistes et protestants pentecôtistes se sont détériorés ainsi progressivement

20. La Messa beat est un courant apparu en Italie dans les années 1960. Ancrées dans le renouveau postconciliaire, les messes beat se caractérisent par l'intrusion de musiques rock associées à des thèmes chrétiens dans l'office liturgique. L'exemple le plus représentatif est sans doute La Messa dei Giovani, composée par Marcello Giombini en 1966. 
dès le milieu des années 1970, entraînant l'abandon définitif du terme " pentecôtiste » pour qualifier les catholiques du Renouveau postconciliaire.

En Égypte, les rapports entre protestants évangéliques et charismatiques catholiques sont plus cordiaux. Une forme de curiosité bienveillante se manifeste bien souvent entre chaque partie, au moins de la part des fidèles. Les croisements et rencontres s'avèrent constants. Ainsi, comme dans les communautés de l'Emmanuel et du Chemin, toutes les obédiences assistent aux offices protestants, de même qu'aux réunions de prières. Ces dernières sont bien souvent organisées par Sameh Maurice, révérend de l'église Qașr al-Dubāra du Caire, accompagné de son groupe de musique, Better Life. Par exemple, le rassemblement du 11 novembre 2011, réunion de prières nocturnes pour l'Égypte, a réuni nombre de chrétiens égyptiens - les églises du Muqațtam étaient bondées -, et pas seulement des coptes. Melkites, coptes-catholiques, catholiques latins, et même, coptesorthodoxes, ont participé à cet événement. Beaucoup se sont également rendus au rassemblement d'Hurghada, organisé pendant deux jours, les 10 et 11 février 2014, notamment par Sameh Maurice et le chanteur copte-orthodoxe Māhir Fāyiz, sous l'intitulé « Pour l'amour de l'Égypte. Un cri du cœur » (Fī ḅubb Miṣr, șarhạa min al-qalb ${ }^{21}$. Ces manifestations peuvent être suivies par tous, car systématiquement retransmises sur la chaîne satellitaire chrétienne Sat7. Quelle que soit leur obédience, la grande majorité des fidèles connaissent fort bien les offices de Sameh Maurice, et y assistent souvent avec enthousiasme.

Il en est de même des réunions de prière organisées par Māhir Fāyiz et son groupe al-Kārūz, en l'église Kanīsat Allāh à Choubra. Tous les samedis soirs, dès 19 h, Māhir Fāyiz se "produit » accompagné de son 'ūd électrique et de ses musiciens, les mêmes que l'on retrouve dans le groupe Better Life. Lui-même copte-orthodoxe, il prêche dans une veine toute charismatique en des termes simples, qui se veulent percutants, en cherchant à provoquer une émotion spirituelle dosée et sincère. Les chants, retransmis en karaoké sur grand écran, dévoilent eux-aussi une simplicité lexicale, des thèmes récurrents et l'usage fréquent de la 'āmmiyya, le dialecte arabe égyptien. Cette question de l'usage de la langue dans les tarnìm-s est prépondérante. Les chants de l'Emmanuel, comme ceux du Chemin Néocatéchuménal, sont des traductions. Ils sont écrits en fusḥā, en arabe classique. Chez Māhir Fāyiz, comme dans les autres groupes de musique religieuse égyptiens - non issus, contrairement à l'Emmanuel et au Chemin, d'Europe -, la 'āmmiyya est préférée à la fusḥā. Elle n'est pas exclusive, mais bien privilégiée.

Le recours au dialecte est valorisé dans un livre intitulé Mahārāt al-Tasbīh (Talents de la louange), publié par le groupe Praise Team of Egypt, également

21. Sur le rôle politique des tarnìm-s, $c f$. Ramzy, 2014. Notons que cet événement se tient en pleine campagne présidentielle. Or l'intitulé, $F \bar{\imath}$ ḅubb Miṣr, était aussi le slogan récurrent des affiches de la campagne d'Al-Sisi, slogan qu'on peut encore lire de nos jours dans les rues, notamment à l'occasion des élections législatives (mai 2015). 
connu sous le nom arabe de Farīq al-Tasbīh, le Groupe de la Louange. La Praise Team of Egypt se définit comme un groupe de "musique arabe chrétienne » ( $m \bar{u} \bar{s} \bar{q} \bar{\imath}$ ' $\operatorname{arab} \bar{\imath}$ masīh $\bar{\imath})$, qui rassemble les trois communautés religieuses : orthodoxe, protestante, catholique. Elle existe depuis 1982, et se nommait, à l'époque, Farīq al-tasbīh hidmatuhu li-l-rabb, L'équipe de louange au service du Seigneur. Les Enfants de la Praise Team (Ațâl farīq al-tasbīh) et les Jeunes de la Praise Team (Šabāb farīq al-tasbīḥ) ont été respectivement lancés en 1997 et 2009. C'est pour eux que ce livre a été écrit. Ce dernier porte un double intérêt : celui de théoriser la composition d'un genre musical religieux en pleine expansion et celui de prétendre représenter les trois confessions, sans distinction particulière - même si en réalité, l'inspiration évangélique, donc protestante, d'une telle démarche est à peine dissimulée.

Dans cet ouvrage de la Praise Team publié en 2013, le responsable du groupe, Būlos Bošra, a cherché à théoriser les compositions de tarnīm-s. Diffusé dans la grande librairie copte cairote Maktabat Mahabba, à Choubra, il doit aider à l'écriture et à la composition musicale des tarnīm-s destinés aux jeunes et aux enfants, comme l'indique le sous-titre Ta'lîf wa-talḥin tarānìm li-l-šabāb wa-l-aṭ̂āl, " composition textuelle et musicale des tarnìm-s pour les jeunes et les enfants ". Il est organisé en quatre parties. La première est consacrée à l'écriture ; l'accent y est mis sur le genre du texte, son style, la langue. On y apprend comment résoudre des problèmes de rimes, comment construire de percutantes métaphores, comment éviter les "erreurs ». L'usage de la 'àmmiyya y est justement recommandé, et doit permettre la compréhension pour le plus grand nombre, la fusḥa étant alors décrite comme trop complexe et pas assez proche des jeunes. L'usage de l'arabe classique permettrait pourtant une circulation plus aisée des répertoires hors d'Égypte, mais cela ne semble pas être une priorité.

La seconde partie, sur laquelle nous nous attarderons davantage, concerne la composition musicale des tarnìm-s. La musique y est annoncée comme le moyen de délivrer une idée, tout en permettant un contrôle des émotions (Bošra, 2013 : 124-125). En ce sens, elle appuie les mots, soutient le texte et en diffuse le sens. L'accent est mis, tout au long de cette partie, sur la nécessité pour les tarnìm-s, d'être une musique de Dieu, pour Dieu. Pour expliciter cette injonction, l'auteur établit des parallèles qui ne sont pas sans rappeler les théories des musicographes antiques. Cette musique à vocation religieuse est alors assimilée aux sons et aux bruits de la nature, témoins d'un ordre " naturel » ou « cosmique ". Au fil des pages, on comprend que la musique doit permettre, finalement, l'analyse mentaliste, en provoquant une représentation mentale qui, potentiellement, débouche sur de l'émotion. Les mises en garde contre une symbolisation musicale liée au Malin s'immiscent régulièrement dans le texte, avec notamment cette question : comment faire une musique pour Dieu sans connaître soi-même le royaume de Dieu? (p. 128) Nous n'en apprendrons néanmoins pas davantage sur les caractéristiques des musiques « diaboliques » dans cet ouvrage. 
En réalité, après un tel discours sur les sons de Dieu et la nature quasi-divine des tarnìm-s, cette partie peut surprendre par la quantité des influences qu'elle propose pour la composition. L'auteur conseille en effet d'écouter toutes sortes de musiques, pour glaner des éléments d'inspiration. Il suggère de naviguer entre différents styles musicaux, d'insérer des rythmes samba, syncopés, rock, ou encore de reproduire, pour renouveler le style, des valses, des marches, des tangos (p. 150). Il présente la possibilité d'user aussi des maqām-s, les modes arabes, dans la composition (p. 151), tout en proposant d'utiliser également, pour varier, les gammes mineure et majeure, plus proches de la musique de variété occidentale (p. 156-159). Il admet du reste que les échelles les plus utilisées dans les tarnìm-s restent les tonalités occidentales (p. 157). De multiples influences sont ainsi admises.

Des détails plus techniques apparaissent dans les pages qui suivent, sous le titre « Recettes d'une bonne mélodie » (Ṣifāt al-laḥn al-ğayyid, p. 159) : les modulations, la longueur des phrases, les enchaînements d'accords recommandés, l'ambitus ${ }^{22}$ (madā) possible, etc. Quelle que soit la composition, elle doit " parler » aux jeunes, leur être proche. En cela, la composition musicale entre idéalement en correspondance avec des modèles en vogue (p. 215), d'où l'importance de l'usage de la 'āmmiyya. Elle doit également se retenir facilement : nul intérêt, pour le compositeur, de dévoiler son talent à l'aide d'accords sophistiqués. Les harmonies gagneront à être écrites avec simplicité (p. 224).

Comme pour l'Emmanuel et le Chemin, de nombreux tarnīm-s sont composées à partir de versets de la Bible. Il s'agit là, pour B. Bušra, d'un des genres de composition les plus difficiles, notamment parce que cette fois on ne recourt plus à la 'ámmiyya, mais à l'arabe classique, et qu'il convient dès lors de maîtriser le rythme de la prosodie pour pouvoir ensuite composer la mélodie (p. 238).

Après la composition musicale, la troisième partie énumère les qualités nécessaires aux tarnìm-s destinés aux enfants. La quatrième, enfin, propose des pistes pour publier ces nouvelles compositions.

Une volonté d'œuvrer à l'œuménisme se dégage de ces groupes de prières et des communautés charismatiques, aussi bien que de cet ouvrage. Un tel désir d'unité, patent, se retrouve aussi dans les discours des uns et des autres. Il ne s'agit pas, pour les charismatiques comme pour les évangéliques, de se rebeller par rapport à leur paroisse, mais plutôt de trouver, en guise de complément, une aide pour vivre leur foi entre chrétiens au quotidien ${ }^{23}$, quelle que soit l'obédience institutionnellement représentée au sein du groupe.

Du point de vue de l'espace, les chrétiens sont aussi confrontés à cet œcuménisme. Ainsi, le Forum ${ }^{24}$ organisé par les jeunes de l'Emmanuel en Égypte du

22. Distance entre la note la plus grave et la note la plus aiguë de la mélodie.

23. Ce constat est partagé par Gaétan du Roy, 2014 : 357.

24. Rencontres organisées l'été par la Communauté de l'Emmanuel dans une démarche d'évangélisation. Elles s'orientent ainsi autour de la louange, de l'adoration, des enseignements 
20 au 24 août 2014 s'est tenu cette année-là au Karmat al- Wādī, au Wād̄̄ alNatrūn (Nord-Ouest du Caire), qui est une maison consacrée aux retraites, tenue par des coptes-orthodoxes. Ce Forum a réuni plus de trois cents personnes de diverses obédiences, contre environ deux cents en 2013. Pour exister, ces communautés et groupes ont besoin, en Égypte, d'œuvrer les uns avec les autres.

\section{Vers une liberté spirituelle absolue?}

Chez les charismatiques, la musique est assimilée de manière licite à une émotion spirituelle. Les processus d'expression de la foi par la musique suivent même une logique communautaire. Cette logique peut se définir par une nécessité, pour les "frères ", de partager à voix haute leurs prières, leurs doutes, leurs espoirs, en vue d'une libération, d'une ouverture totale et absolue aux autres et à Dieu et, but ultime, d'accéder ainsi à l'Amour et à la Vérité ${ }^{25}$. En ce sens, il s'agit d'une musique qui permet la libération de la prière. Les paroisses d'accueil à la liturgie plus austère et au cadre expressif retenu tolèrent difficilement, voire pas du tout, l'épanchement d'une spiritualité expressive. Néanmoins, à l'extrême inverse, le Renouveau charismatique a souvent été assimilé à un déversement excessif de libertés. Les membres des communautés pouvaient en effet être perçus comme des hordes d'exaltés frénétiques et subissaient parfois l'usage de sobriquets peu flatteurs, comme par exemple celui de « dévisseurs d'ampoules » en France.

En Égypte, cette appréhension devant une émotion spirituelle débordante est bien analysée par Gaétan du Roy, notamment lorsqu'il rapporte les propos de l'Anbā Mūsā, évêque responsable des services sociaux : « [ce dernier] leur reproche [aux évangéliques] de pratiquer des tarnīm-s "du corps et des émotions" (ğasad wa-infi'ālāt) au lieu de ceux "de la raison et de l'esprit" ('aql wa-rūḥ) » (du Roy, 2014 : 347) ${ }^{26}$. Plus récemment, en juin 2014, une motion prononcée par un évêque copte orthodoxe interdisant le recours aux tarnìm-s protestants au sein des églises du Muqațtam a suscité beaucoup de réactions de tous ordres (Id. : 392). Cette crainte d'une débauche musicale est néanmoins de moins en moins perceptible au sein de l'Église catholique, puisque les communautés charismatiques sont désormais reconnues par le Vatican. Les catéchèses de Kiko pour le Chemin Néocatéchuménal, par exemple, ont été approuvées par la Congrégation pour l'évangélisation des peuples en 2011. Avant cela, en 2008, Jean-Paul II avait encouragé l'existence du Chemin, en admettant notamment son utilité en tant

et des discussions ; tous ceux qui le souhaitent peuvent y participer, peu importe leur confession. Le premier Forum en Égypte s'est tenu en 2009, lancé sous l'impulsion de Yasmine Nāder.

25. "Amour et vérité » est un des leitmotiv de la Communauté de l'Emmanuel, sur lequel se fonde régulièrement la réflexion religieuse des membres lors de séminaires tenus par des «frères ". L'expression est issue du Psaume 85.

26. Au sujet de réactions hostiles aux tarnìm-s utilisés en contexte charismatique et évangélique, Gaétan du Roy remarque encore que «l'appel à la raison se situe du côté du respect de la tradition tandis que les émotions [...] sont assimilées au désordre » (du Roy, 2014 : 347). 
qu' « œuvre pour la nouvelle évangélisation ${ }^{27}$ ». Cette reconnaissance renforce le caractère licite des pratiques musicales et des chants communautaires.

Enfin, rappelons que ces communautés prennent appui sur le rôle et la responsabilité des laïcs. Ces derniers doivent pouvoir s'exprimer davantage, en priant à haute voix, en dirigeant les fidèles, et en proposant des réflexions liées à la théologie ou à des règles de vie lors de séminaires. Le rôle de la louange, prépondérant, s'appuie précisément sur le laïc et sur sa capacité nouvelle à intercéder luimême auprès de Dieu. Les chants de louange sont alors courts, faciles à retenir, percutants, accessibles à tous. Ils laissent libre cours à l'improvisation (chant glossolale) et permettent de chanter facilement les harmonies. Dans le cadre de cette spiritualité libérée, comme dans l'Emmanuel, ceux qui dirigent la prière de la louange sont des laïcs, donc de simples membres de la communauté. En outre, ces compositions de louange et d'adoration permettent de chanter, bouger, lever les mains, ... ce qui n'est absolument pas le cas des chants liturgiques byzantins ou coptes, pour ne citer qu'eux. Dans ce contexte, la musique vise une libération spirituelle plus systématique.

Dans une précédente réflexion (Gabry-Thienpont, 2014a), j’avais envisagé les tarnìm-s comme une seule et même catégorie de chants, réunis au sens large sous la bannière du paraliturgique. Nous pouvons à présent entrevoir l'existence de nombreuses sous-catégories de tarnīm-s, aux influences stylistiques multiples. Dans le cas de la Communauté de l'Emmanuel, ce sont prioritairement la louange et l'adoration qui sont soutenues par les compositions musicales, considérées comme «l'une des manifestations de l'Esprit Saint (Goursat, 2011 : 188). Ces chants, à l'origine des compositions françaises, sont de plus en plus fréquemment égyptianisés. Dans le Chemin Néocatéchuménal, les accords de Kiko à la guitare occupent les célébrations et donnent à entendre une parole rythmée, aux couleurs gypsies et orientales. Ces chants visent alors la diffusion et la compréhension du kérygme.

Chez les évangéliques égyptiens, les influences et les objectifs sont plus foisonnants et moins marqués régionalement. Les compositions de la Praise Team of Egypt, de Better Life et de Māhir Fāyiz et son groupe, Farīq al-Kārūz, s'illustrent par des échelles arabes, mais pas uniquement, appliquées à des rythmes jazz et blues, le tout globalement lent, rappelant autant les compositions du prêtre libanais Mansour Labaki ${ }^{28}$ que certains tubes de chanteurs égyptiens pop' à la mode,

27. Lettre du pape Jean-Paul II intitulée « Ogni Qualvolta », 30 août 1990, consultée le 6 juillet 2014 : http://www.vatican.va/holy_father/john_paul_ii/letters/1990/documents/hf_jpii_let_19900830_ogni-qualvolta_it.html

28. Pour un exemple de ces types de chant:

Mansour Labaki : https://www.youtube.com/watch?v=A4oWcTzSOfQ, vidéo consultée le 2 juillet 2014.

Māhir Fāyiz: https://www.youtube.com/watch?v=Tl3e5NV8UNE, vidéo consultée le 2 juillet 2014. 
comme Haytam Šāker. Dans le cas de Māhir Fāyiz, l'usage du 'ūd entérine définitivement la signature orientale de ses tarnìm-s. Ce point revêt une certaine importance : en dépit de leurs origines occidentales, ces communautés en Égypte sont à présent largement investies par des individus qui éprouvent le besoin d'assimiler leur identité nationale à leur foi. La musique devient ainsi un moyen de marquer ce lien.

Il convient néanmoins de ne pas surestimer l'impact des communautés charismatiques dans l'Égypte d'aujourd'hui. Loin d'être majoritaires, ces mouvements permettent de contourner la très forte cléricalisation de l'Église copte et d'offrir une forme d'expression plus spontanée, plus libre. Mais si d'aucuns y voyaient peut-être les prémices d'un délitement du lien communautaire copte, en réalité, pour l'instant du moins, il n'en est rien. Chaque «frère » continue en effet de fréquenter sa paroisse originelle, ce qui signifie que chacun reste attaché à une structure ecclésiale classique. Nader, musicien responsable des chants au sein du Chemin Néocatéchuménal, par exemple, en plus d'être un guitariste hors-pair, fait partie de la chorale de sa paroisse grecque-catholique, où les mélodies liturgiques sont a cappella. Sa voix de ténor y est du reste fort appréciée. L'adhésion au Chemin - et aux activités hebdomadaires que cela suppose - ne l'empêche pas de mener une vie liturgique régulière au sein de sa paroisse, bien au contraire. Le Renouveau charismatique des catholiques en Occident s'est ainsi immiscé en Égypte et traduit une volonté de renouvellement de la manifestation de la foi, notamment par la musique. Arrivé peu de temps après les débuts du Renouveau copte, cet autre renouveau occupe désormais une place non négligeable au sein de l'espace liturgique et y rencontre un succès grandissant.

\section{Séverine GABRY-THIENPONT \\ Centre de Recherche en Ethnomusicologie - CNRS \\ IFAO, Le Caire \\ sevgabry@free.fr}

\section{Bibliographie}

ArgüEllo Kiko, 2013, Le Kérygme. Dans les baraques avec les pauvres, Paris, Cariscript. Aubourg Valérie, 2014, "Chant céleste : la glossolalie en milieu pentecôtiste charismatique à l'Île de la Réunion ", Anthropologie et sociétés 38/1, p. 245-262.

Bušra Būlos, 2013, Muhārāt al-Tasabị̣̄ 2, tā’līf w-talḥ̄n tarānīm lil-šabāb w-al-āṭāl, Le Caire, Farīq al-tasabīh.

Csordas Thomas J., 1997, Language, Charisma, and Creativity: The Ritual Life in a Religious movement, Berkeley, Los Angeles, London, University of California Press.

Du Roy Gaétan, 2014, Le prêtre des chiffonniers ou la construction d'une autorité religieuse au Caire, entre charisme, tradition et clientélisme (1974-2014), thèse de doctorat, Université catholique de Louvain.

EL-Khawaga Dina, 1993, Le Renouveau copte. La communauté comme acteur politique, thèse de doctorat, Paris, IEP. 
GabRY-THIEnPont Séverine, 2013, Anthropologie des musiques coptes en Égypte contemporaine, thèse de doctorat, Nanterre, Université Paris Ouest Nanterre la Défense.

-, 2014a, "Tarânîm et madîh : chants liturgiques coptes ou chansons populaires ?", Cahiers Rémois de Musicologie 7, p. 87-99.

-, 2014b, " Dire ou chanter les chants coptes en Égypte contemporaine », in Anne DamonGuillot et Béatrice Ramaut-Chevassus, Dire/Chanter : passages, Publications de l'Université de Saint-Étienne, p. 273-289.

Goursat Pierre, 2011, Paroles, rassemblées et présentées par Martine Catta, Paris, Éditions de l'Emmanuel.

Hamilton Alastair, 2006, The Copts and the West, Oxford, Oxford University Press.

Heyberger Bernard, 2001, Hindiyya : mystique et criminelle (1720-1798), Paris, Aubier.

LANDRON Olivier, 2004, Les communautés nouvelles. Nouveaux visages du catholicisme français, Paris, Éditions du Cerf, coll. "Histoire ".

Laurentin René, 1974, Pentecôtisme chez les catholiques, risques et avenir, Paris, Éditions Beauchesne.

Legrand Hervé, 1962, "Le Renouveau copte », Istina 2, p. 133-150.

Martin Maurice, 1990, "Les coptes catholiques, 1880-1920 », Proche-Orient Chrétien 40, p. 33-55.

Peyrous Bernard, Catta Martine, 2006, Le feu et l'espérance, Paris, Éditions de l'Emmanuel.

PietTe Albert, 2014, "Quand croire, c'est faire, et un peu plus ", in Emma AubinBoltanski, Anne-Sophie Lamine et Nathalie Luca (dir.), Croire en actes. Distance, intensité ou excès ?, Paris, L'Harmattan, p. 63-76.

PINA Christine, 2001, Voyage au pays des charismatiques, Paris, les Éditions de l'Atelier.

Ramzy Carolyn M., 2014, "To Die is Gain: Singing a Heavenly Citizenship among Egypt's Coptic Christians ", Ethnos: Journal of Anthropology, p. 1-22.

Sedra Paul, 2011, From Mission to Modernity: Evangelicals, Reformers and Education in Nineteenth-Century Egypt, Londres, New York, I.B. Tauris.

SHARKeY Heather, 2008, American Evangelicals in Egypt: Missionary encounters in an age of Empire, Princeton, Princeton University Press.

Willaime Jean-Paul, 1999, « Le Pentecôtisme : contours et paradoxes d'un protestantisme émotionnel ", Archives des sciences sociales des religions, nº 105, p. 5-28.

\section{Musiques et charismes chez les chrétiens en Égypte au début du xxl siècle. L'exemple catholique.}

En Égypte, l'engouement exponentiel pour les musiques paraliturgiques traduit une implication grandissante de la jeunesse, toutes obédiences chrétiennes confondues, au sein de groupes religieux charismatiques et évangéliques. Après le Renouveau copte de la deuxième moitié $d u X^{e}$ siècle, c'est au tour du Renouveau charismatique catholique postconciliaire d'investir les paroisses égyptiennes. Les communautés occidentales créées dans la lignée du concile Vatican II sont de mieux en mieux représentées en Égypte, même si cette représentation se cantonne à la capitale. Dans ces groupes, chants et musiques occupent une place centrale, et participent ainsi d'un renouvellement de la foi chrétienne en Égypte.

Mots-clés : Égypte, musique, paraliturgique, renouveau charismatique, copte. 


\section{Music and Charisma Among the Christians in Early $21^{\text {st }}$ Century Egypt. The Catholic Case}

In Egypt, the exponential enthusiasm for paraliturgical music reflects an increasing involvement of Christian youth of all faiths within charismatic and evangelical religious groups. After the Coptic Renewal that took place during the second part of the twentieth century, it is now the turn of the Catholic Charismatic Renewal to perform within Egyptian parishes. Communities from the USA and Europe, which developed in line with the Second Vatican Council, are being more than ever represented in Egypt, even if this representation is only noticeable in the Egyptian capital. Within these groups, songs and music play a central role, and participate in the renewal of the Christian faith in Egypt today.

Key words: Egypt, music, paraliturgical, Charismatic Renewal, Copt.

\section{Músicas y carismas entre los cristianos en Egipto a principios del siglo XXI. El ejemplo católico}

En Egipto, el entusiasmo exponencial por las músicas paralitúrgicas traduce una implicación creciente de la juventud de todas las ramas cristianas en el seno de los grupos religiosos carismáticos y evangélicos. Después de la Renovación copta de la segunda mitad del siglo XX, la Renovación Carismática católica posconciliar intenta apropiarse de las parroquias egipcias. Las comunidades occidentales creadas en la senda del Concilio Vaticano II están cada vez más representadas en Egipto, aunque esta representación se limite a la capital. En estos grupos, cantos y música ocupan un lugar central, participando asi de una renovación carismática copta.

Palabras clave: Egipto, música, paraliturgia, renovación carismática, coptos. 
